\title{
Ultrasonography in psoriatic disease
}

\section{Mihaela Elena Marina*1, Carolina Botar Jid*2, Iulia Ioana Roman³, Carmen Mihaela Mihu, Alexandru Dumitru Tătaru ${ }^{4}$}

\author{
${ }^{1}$ Histology Department, ${ }^{2}$ Radiology Department, ${ }^{3}$ Physiology Department, ${ }^{4}$ Dermatology Department, "Iuliu Haţieganu" \\ University of Medicine and Pharmacy, Cluj-Napoca, Romania \\ * the authors shared the first authorship
}

\begin{abstract}
Psoriasis represents a common, chronic inflammatory disease involving the skin, nails and joints. Nail psoriasis in usually investigated and diagnosed by clinical examination. New insights support ultrasonography as being a non-invasive reliable imaging technique for studying nail and skin involvement in patients with psoriasis. Power Doppler frequency, higher than $10 \mathrm{MHz}$ enables a very sensitive detection and semiquantitative assessment of the blood flow at the dermal level and in the nail bed. In this review we will present the main US findings in skin and nail psoriasis, and discuss the importance of ultrasonography in diagnosing and monitoring psoriasis vulgaris.
\end{abstract}

Keywords: nail, skin, psoriasis, ultrasonography, power Doppler.

\section{Introduction}

Psoriasis is a chronic, relapsing skin disease affecting millions of people worldwide, with a general prevalence estimated to be approximately $2-3 \%$ [1]. This complex skin disorder has an immune-mediated and multifactorial determination involving both genetic and environmental factors. Prototype illness of chronic dermatoses, psoriasis is a systemic inflammatory disease with an immunologic mechanism that combines dermic inflammation with secondary epidermic hyperplasia [2].

Diagnosis of psoriasis is based on clinical history and physical examination, the histological examination remaining the standard reference for the final diagnosis [3]. Although the skin has the most characteristic findings of psoriasis, other organs, such as nails and joints, may be also affected in a great number of patients. For describ-

Received 30.05.2015 Accepted 29.06.2015

Med Ultrason

2015, Vol. 17, No 3, 377-382

Corresponding author: Mihaela Elena Marina

Histology Discipline, "Iuliu Haţieganu"

University of Medicine and Pharmacy,

6-8 Louis Pasteur street

400349 Cluj Napoca, Romania

Phone: +4 0740075805

E-mail: marina.elena@umfcluj.ro ing the entire spectrum of psoriasis clinical features, the designation "psoriatic disease" has been suggested [4].

As reported by Van Laborde et al there is a $80 \%$ to $90 \%$ lifetime incidence of nail involvement in psoriatic patients [5]. Nail psoriasis is usually diagnosed only by physical examination, observed features depending on nail matrix or nail bed involvement. The presence of nail psoriasis is clinically relevant as patients often complain of pain, functional impairment and social burden, resulting in a significant restriction of daily activities and quality of life [6-8].

Cases with only nail psoriasis are quite problematic because they often require nail biopsy, a painful procedure that may cause detrimental cosmetic permanent changes, and should only be used when diagnosis cannot be made by other means [9].

High frequency ultrasound (HFUS) represents a noninvasive imaging method which allows viewing the anatomical and physiologic details of the skin and nails and provide the morphological appearance of the skin and nail lesions, together with changes of the underlying tissue [10].

In this review we will describe the potential of ultrasonography using high frequency probes and power Doppler technique in depicting psoriasis induced skin and nail changes. The images exemplified in this report are rep- 
resentative examples of the ability of sonography to evidence and describe minimal morphostructural and blood flow changes in both psoriatic plaques and onychopathy.

\section{Technical considerations}

In order to provide optimal visualization for skin and nail, in ultrasound examination, high or very high frequency $(15-40 \mathrm{MHz})$ linear broad bandwidth transducers are used. Using transducers with very high frequency characterized lesions in the submillimeter range (down to $0.1 \mathrm{~mm}$ ) can be identified. Gray-scale ultrasound (2D) allows a good characterization of the changes in the skin or in the nails [10]. Doppler ultrasound allows visualization of the blood vessels with details about the presence of vascularization, the normal or abnormal appearance, the flow direction and velocity.

The images we provide in this review were taken by an experienced sonographer using a Ultrasonix System equipped with a variable-frequency transducer ranging from $20-40 \mathrm{MHz}$ to visualize nail and skin anatomy. In order to observe blood flow we used a Hitachi EUB 8500 System equipped with a variable-frequency transducer ranging from $6.5-13 \mathrm{MHz}$.

Detailed study of skin and nails structure requires the use of high frequency transducers. As known, increasing the frequency will decrease the wavelength which will result in increasing the axial resolution of the ultrasound image. The ultrasound beam is narrowed at that depth revealing the best lateral resolution which improves the image quality and ensures high definition of tissue.

Both normal and psoriatic skin and nail lesions are first scanned in the gray scale mode to detect morphostructural changes and followed by power Doppler technique to detect blood flow in the dermis and nail bed, respectively.

Vascularization in psoriatic plaque was assessed using power-Doppler ultrasound (PD) paying attention not to apply pressure on the tissues under investigation and avoiding the "blanching" of PD signal due to compression by the transducer [11].

Due to the fact that normal thickness of the skin is widely variable according to the anatomic area, it is rational to perform an ultrasound comparison between the psoriatic plaque and the normal surrounding skin in the same area of the same patient. Guastalla et al suggested normal sonographic values of thickness in the different layers of the skin [12]. Ultrasonography may be useful to discriminate between inflammatory, vascular or tumoral pathology, not only for diagnosis, but also for monitoring the evolution under therapy and administered therapy efficiency assessment [10].

\section{Ultrasonography of the healthy skin}

The skin consists of two layers: epidermis and dermis, but due to its proximity and frequent involvement in skin pathology, the subcutaneous tissue or hypodermis can be viewed as the third layer. Each of these three layers has a different sonographic appearance depending on its main components.

In normal non-glabrous skin, due to the presence of keratin, the epidermis appears as a thin, continuous hyperechoic line with homogeneous thickness or as a bilaminar hyperechoic and parallel lines in the glabrous skin of the palmar and plantar regions. Containing a large amount of collagen fibers the dermis is visualized as a hyperechoic and homogenuous band, but less bright than the epidermis, whereas the subcutaneuous tissue (with fat lobules) is characteristically hypoechoic with hyperechoic lines generated by fibrous septa of connective tissue [13-15]. Arterial and venous vessels located subcutaneous appear as thin anechoic tubes, while the bone surface appears as a separate hyperecohoic line (fig 1a) [10].

\section{Ultrasonography of the healthy nail}

The nail unit is comprised of the nail plates (dorsal and ventral), nail bed, ungual matrix, periungual tissues (proximal and lateral nail folds), and the hyponychium.

On ultrasound, the components of the nail unit are clearly visible: the dorsal and ventral nail plates appear as bilaminar parallel hyperechoic lines (due to the highly keratinous component) with a virtual hypoechoic space in between. The nail bed has a hypoechoic band appearance, not clearly distinguishable from the underlying hypodermis, and usually turning slightly hyperechoic in the proximal region beneath the ungual matrix. The nail folds have ultrasonographic characteristics resembling to those of the nonglabrous skin, although with less fat lobules in the subcutaneous tissue [16-17]. A continuous hyperechoic line below the nail bed corresponds to the dorsal bony margin of the distal phalanx (fig 1b). Doppler color and power Doppler ultrasound can detect the presence of low-velocity blood flow within arterial and venous vessels in the nail bed (fig 2) $[10,13,18]$.

\section{Psoriatic plaque}

In psoriatic plaque, the changes are located both in the epidermis and dermis, without involvement of the underlying hypodermis. The sonographic features of the psoriatic plaque are easy to distinguish from the surrounding healthy skin. As a result of increased keratinocyte proliferation in the epidermis and abnormal concentration of 


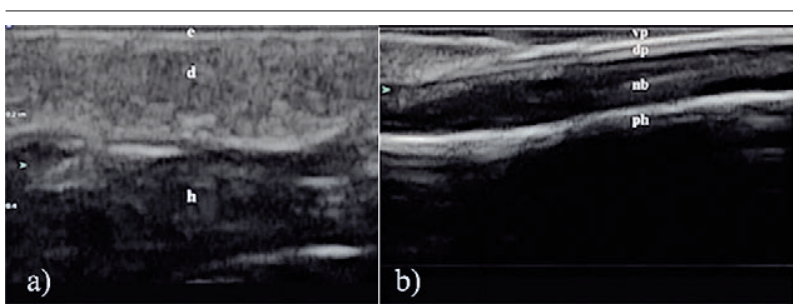

Fig 1. a) Normal appearance of the skin - gray scale ultrasound (e-epidermis, $\mathrm{d}$ - dermis, $\mathrm{h}$ - hypodermis); b) Normal appearance of the nail - 2D ultrasound (vp - ventral plate, dp - dorsal plate, $\mathrm{nb}$ - nail bed, $\mathrm{ph}$ - bone surface of the distal phalanx)

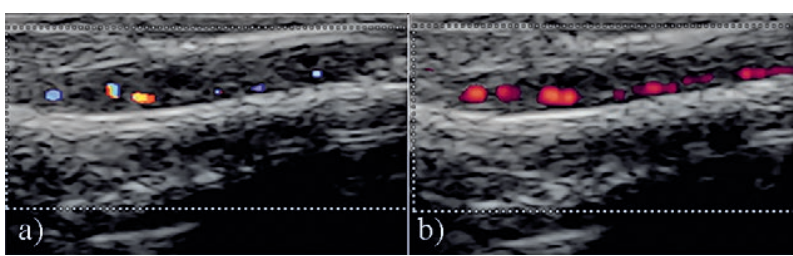

Fig 2. Normal vascularization of the nail: a) color Doppler; b) power Doppler

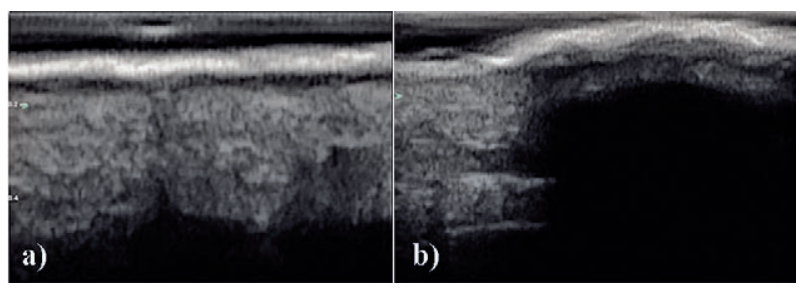

Fig 3. a) Thickening of the epidermis in a psoriatic plaque; b) posterior acoustic shadowing artifact in a case of psoriatic plaque

proinflammatory cells in the dermis, these two skin layers appear thicker than normal surrounding skin when using grey-scale ultrasound examination. When marked thickening of the epidermis occurs there may be seen a posterior acoustic shadowing artifact, that impedes/obstructs a clear assessment of the underlying dermis (fig 3) [19-22]. HFUS of a psoriatic plaque reveals a three-layer structure: a hyperechoic band that represents epidermal parakeratotic scaly superficial layer and suprapapillary epidermis, a non-echoic subepidermic band that corresponds to elongated epidermal rete ridges with congested and edematous loose connective tissue and, in the lower part, the reticular dermis appears as a hyperechoic band [19].

Although not specific for psoriasis, a hypoechoic band in the upper dermis, corresponding to inflammatory edema and vasodilatation, was a commonly observed sonographic feature $[23,24]$ and reported as being a reliable indicator of active stages of the disease. Other inflammatory conditions such as acantoma, atopic dermatitis or contact dermatitis can also present this hypoechoic dermal band [23].
Ultrasound indicators of effective therapy, as described on gray scale mode, were the reduction in thickness of both epidermal and dermal layers, and mainly the disappearance of the hypoechoic band in the superficial dermis $[24,25]$.

Ultrasonographic monitoring of the activity and severity of psoriatic involvement has been the objective in several investigations in patients affected by this invalidating disease [26,27]. Skin high-frequency ultrasonography may be used in evaluating local treatment response. Lacarrubba et al in a study on 30 patients using topical clobetasol propionate $0.05 \%$ foam, reported a reduction in psoriatic plaque thickness when monitoring treatment response with a 20-MHz HFUS [28].

Depending on the severity in the active inflammatory phase, an increased blood flow signal within the dermis of the psoriatic plaque can be detected by color or power Doppler. A wide spectrum of dermal microvascular changes were reported: elongated, dilatated, and twisted capillary loops $[19,29]$.

As reported, color and power Doppler ultrasound provides useful and valid assessment in short-term monitoring of disease activity and treatment efficacy [30]. Gutierrez et al found a significant correlation between power Doppler findings and both the extent of skin involvement and histopathological degree of vascularization before and after etanercept treatment. These data advocate in favor of validation power Doppler findings when assessing dermal perfusion changes in patients with plaque psoriasis [31].

Ultrasonography in association with clinical examination was reported as useful in evaluating the therapeutic efficacy of oral cyclosporine in 20 patients with plaque psoriasis, the capillaroscopic vascular response being monitored through videodermatoscopy in order to detect early relapsing signs [32].

\section{Psoriatic nail}

In psoriatic onychopathy the ultrasonographic morphologic changes are present in both dorsal and ventral nail plates, and also in the nail bed. Going from early to late nail psoriasis phases the ultrasound features are represented by the thickening of the nail bed (measured as the distance between the ventral plate and the bony margin of the distal phalanx), poorly defined ventral plate with focal hyperechoic areas, and thickening of both dorsal and ventral plates with a wavy-shaped or convex nail appearance. Initially the dorsal nail plate may show loss of definition and small hyperechoic areas, while in evolution irregular undulation and superficial depressions appear $[13,33]$. Using the color or power Dop- 


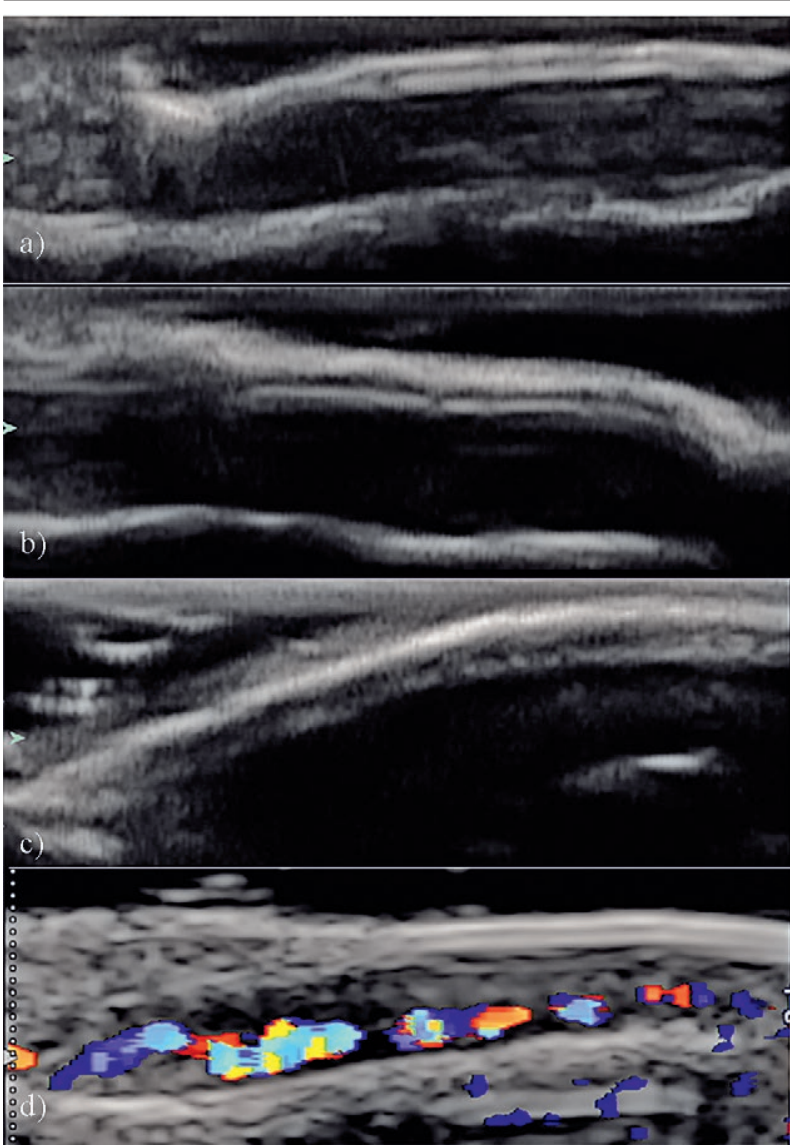

Fig 4. Deletion of the space between the dorsal and ventral plate (a) in psoriatic nails; b) dorsal nail plate irregularities; c) convex nail appearance; d) enlarged and tortuous nail bed blood vessels

pler technique an increased blood flow can be detected within the nail bed, due to the tortuous, enlarged blood vessels (fig 4).

As reported by Gutierrez in a study on 30 patients with psoriasis compared with 15 healthy participants, an increased blood flow both in the dermis and the nail bed can be detected on active phases of onychopathy using power Doppler ultrasonography [13].

A modified blood supply in the nail unit of patients with psoriasis was reported when assessing the Nailfold Vascularity Resistance Index (NVRI). Compared with healthy subjects, patients with psoriasis have a higher resistance to blood flow. The presence of nail involvement in patients with psoriasis was associated with elevated NVRI than in patients with psoriasis with no nail involvement. Husein El-Ahmed et al investigated the vascular resistance in nail vessels in 22 patients with psoriasis and found a higher resistance of the blood flow in psoriatic patients compared with healthy subjects [34]. Future studies on a larger series of patients and in cor- relations with histological data are warranted in order to support these observations.

\section{Psoriatic arthropathy}

Psoriatic arthropathy, the major systemic inflammatory complication of psoriasis, occurring in $5-30 \%$ of patients with cutaneous lesions [3], involves axial skeleton and peripheral joints with a progressive and debilitating evolution. The initial sites of inflammation are the entheses, the Achilles tendon being the first to be affected. In a study conducted on 59 patients with chronic plaque psoriasis Pistone et al reported that ultrasonography of the Achilles tendon can detect enthesitis before clinical signs or symptoms and suggested the use of this technique in the early diagnosis of psoriatic arthropathy [35].

When compared to clinical examination in assessing both disease activity and articular involvement in psoriatic arthritis, ultrasonography proved to be a sensitive method and provided complementary information to radiography and magnetic resonance imaging $[36,37]$.

Studies in psoriasis cohorts including patients without clinical evidence of psoriatic arthritis, indicated a high prevalence of synovitis and enthesis damage [38,39]. In a study comparing patients with psoriasis without musculoskeletal disease to patients with other skin diseases and no musculoskeletal disease, Naredo et al reported ultrasonography synovitis signs in $3.2 \%$ of joints and $1.3 \%$, respectively [40].

In patients with clinically classified early psoriatic oligoarthritis gray-scale and power Doppler ultrasonography detected subclinical synovitis in more joints than the clinical examination, allowing patients to be reclassified as having polyarthritis, as reported by Freeston et al [41].

Due to the close anatomic connection between the nails and extensor digitorum tendon attachment to the bone and the periosteum, the higher prevalence of nail involvement in patients with psoriatic arthritis could be explained [42]. Recent studies underlined the existence of a link between nail psoriasis and synovio-entheseal complex in psoriatic arthritis [43-45]. Therefore the presence of nail changes in patients with distal interphalangeal joint involvement could be a clinical predictor of psoriatic arthritis and patients should be further investigated in order to enhance a precocious diagnosis [46,47].

Nail involvement in psoriasis causes psychosocial and aesthetic impairments, and is a predictor for more severe psoriasis and a higher risk for developing psoriatic arthritis [48]. 


\section{Conclusions}

Ultrasound examination represents an important non invasive imaging technique in the assessment of both psoriatic plaque and nail involvement in psoriasis vulgaris. Ultrasound can provide accurate information regarding the anatomy of the nail, nail bed and nail plates, and the Doppler technique enables dermis and nail bed blood supply assessment. Having no risk of irradiation, ultrasound can be used in diagnosing and long term treatment follow-up in psoriasis vulgaris. Studies aiming at investigating diagnostic value, validity issues including accuracy, and reproducibility are required to sustain the role of ultrasonography in evaluating skin and nail psoriasis induced modifications and to define the impact of these US findings in daily clinical practice.

\section{Conflict of interest: None}

Acknowledgement: This paper was published under the frame of the European Social Fund, Human Resources Development Operational Programme 2007-2013, project no. POSDRU/159/1.5/138776

\section{References}

1. Lima XT, Minnillo R, Spencer JM, Kimball AB. Psoriasis prevalence among the 2009 AAD National Melanoma/Skin Cancer Screening Program participants. J Eur Acad Dermatol Venereol 2013; 27: 680-685.

2. Parisi R, Symmons D, Griffiths C, Ashcroft DM; Identification and Management of Psoriasis and Associated ComorbidiTy (IMPACT) project team. Global epidemiology of psoriasis: a systematic review of incidence and prevalence. J Invest Dermatol 2013; 133: 377-385.

3. Nestle FO, Kaplan DH, Barker J. Psoriasis. N Engl J Med 2009; 361: 496-509.

4. Scarpa R, Ayala F, Caporaso N, Olivieri I. Psoriasis, psoriatic arthritis, or psoriatic disease? J Rheumatol 2006; 33: 210-212.

5. Van Laborde S, Scher RK. Developments in the treatment of nail psoriasis, melanonychia striata, and onychomycosis. A review of the literature. Dermatol Clin 2000; 18: 37-46.

6. Gupta AK, Cooper EA. Psoriatic nail disease: quality of life and treatment. J Cutan Med Surg 2009; 13(Suppl 2): S102-S106.

7. Reich A, Szepietowski JC. Health-related quality of life in patients with nail disorders. Am J Clin Dermatol 2011; 12: 313-320.

8. Baran R. The burden of nail psoriasis: an introduction. Dermatology 2010; 221(Suppl 1): 1-5.

9. Grover C, Reddy BS, Uma Chaturvedi K. Diagnosis of nail psoriasis: importance of biopsy and histopathology. Br J Dermatol 2005; 153: 1153-1158.
10. Wortsman X, Wortsman J, Carreño L. Sonographic Anatomy of the Skin, Appendages, and Adjacent Structures. In: Wortsman X, Jemec GBE. Dermatologic Ultrasound with Clinical and Histologic Correlations. New York, Springer 2013: $15-38$

11. Joshua F, de Carle R, Rayment M, et al. Power Doppler 'blanching' after the application of transducer pressure. Australas Radiol 2005; 49: 218-221.

12. Guastalla P, Guerci VI, Fabretto A, et al. Detection of epidermal thickening in GJB2 carriers with epidermal US. Radiology 2009; 251: 280-286.

13. Gutierrez M, Wortsman X, Filippucci E, De Angelis R, Filosa G, Grassi W. High-frequency sonography in the evaluation of psoriasis: nail and skin involvement. J Ultrasound Med 2009; 28: 1569-1574.

14. Wortsman X, Wortsman J. Clinical usefulness of variablefrequency ultrasound in localized lesions of the skin. J Am Acad Dermatol 2010; 62: 247-256.

15. Wortsman X, Jemec GB. Common inflammatory diseases of the skin: from the skin to the screen. Adv Psor Inflamm Skin Dis 2010; 2: 9-15.

16. Wortsman X, Jemec GB. Ultrasound imaging of nails. Dermatol Clin 2006; 24: 323-328.

17. Cechini A, Montella A, Ena P, Meloni GB, Mazzarello V. Ultrasound anatomy of normal nails unit with $18 \mathrm{MHz}$ linear transducer. Ital J Anat Embryol 2009; 114: 137-144.

18. Wortsman X, Wortsman J, Soto R, et al. Benign tumors and pseudotumors of the nail: a novel application of sonography. J Ultrasound Med 2010; 29: 803-816.

19. Gupta AK, Turnbull DH, Harasiewicz KA, et al. The use of high-frequency ultrasound as a method of assesing the severity of a plaque of psoriasis. Arch Dermatol 1996; 132: 658-662.

20. Wortsman X, Carreño L, Morales C. Inflammatory Diseases of the Skin. In: Wortsman X, Jemec GB. Dermatologic Ultrasound with Clinical and Histologic Correlations. New York: Springer, 2013: 73-118.

21. Olsen LO, Serup J. High-frequency ultrasound scan for non-invasive cross-sectional imaging of psoriasis. Acta Derm Venereol 1993; 73: 185-187.

22. El Gammal S, Auer T, Popp C, et al. Psoriasis vulgaris in $50 \mathrm{MHz}$ B-scan ultrasound: characteristic features of stratum corneum, epidermis and dermis. Acta Derm Venereol Suppl (Stockh) 1994; 186: 173-176.

23. Di Nardo A, Seidenari S, Giannetti A. B-scanning evaluation with image analysis of psoriatic skin. Exp Dermatol 1992; 1: 121-125.

24. Vaillant L, Berson M, Machet L, Callens A, Pourcelot L, Lorette G. Ultrasound imaging of psoriatic skin: a noninvasive technique to evaluate treatment of psoriasis. Int $\mathrm{J}$ Dermatol 1994; 33: 786-790.

25. Serup J. Non-invasive quantification of psoriatic plaque: measurement of skin thickness with $15 \mathrm{MHz}$ pulsed ultrasound. Clin Exp Dermatol 1984; 9: 502-508.

26. Wortsman X, Holm EA, Jemec GB, et al. Fifteen-megahertz high resolution ultrasound examination of psoriatic nails. Rev Chil Radiol 2004; 10: 6-9. 
27. Grassi W, Filippucci E. Is power Doppler sonography the new frontier in therapy monitoring? Clin Exp Rheumatol 2003; 21: 424-448.

28. Lacarrubba F, Nardone B, Musumeci ML, Micali G. Ultrasound evaluation of clobetasol propionate $0.05 \%$ foam application in psoriatic and healthy skin: a pilot study. Dermatol Ther 2009; 22(Suppl. 1): S19-S21.

29. Wortsman X, Jemec GB. Ultrasound imaging of the nails. Dermatol Clin 2006; 24: 323-328.

30. Gutierrez M, Filippucci E, Bertolazzi C, Grassi W. Sonographic monitoring of the psoriatic plaque. J Rheumatol 2009; 36: 850-851.

31. Gutierrez M, De Angelis R, Bernardini ML, et al. Clinical, power Doppler sonography and histological assessment of the psoriatic plaque: short-term monitoring in patients treated with etanercept. Br J Dermatol 2011; 164: 33-37.

32. Musumeci ML, Lacarrubba F, Fusto CM, Micali G. Combined clinical, capillaroscopic and ultrasound evaluation during treatment of plaque psoriasis with oral cyclosporine. Int J Immunopathol Pharmacol 2013; 26: 1027-1033.

33. Wortsman X, Gutierrez M, Saavedra T, Honeyman J. The role of ultrasound in rheumatic skin and nail lesions: a multi-specialist approach. Clin Rheumathol 2011; 30: 739-748.

34. Husein El-Ahmed H, Garrido-Pareja F, Ruiz-Carrascosa JC, Naranjo-Sintes R. Vessel resistance to blood flow in the nailfold in patients with psoriasis: a prospective case-control echo Doppler-based study. Br J Dermatol 2012; 166: 54-58.

35. Pistone G, La Vecchia M, Pistone A, Bongiorno MR. Achilles tendon ultrasonography may detect early features of psoriatic arthropathy in patients with cutaneous psoriasis. Br J Dermatol 2014; 171: 1220-1222.

36. Wiell C, Szkudlarek M, Hasselquist M, et al. Ultrasonography, magnetic resonance imaging, radiography, and clinical assessment of inflammatory and destructive changes in fingers and toes of patients with psoriatic arthritis. Arthritis Res Ther 2007; 9: R119.

37. Weiner SM, Jurenz S, Uhl M, et al. Ultrasonography in the assessment of peripheral joint involvement in psoriatic arthritis: a comparison with radiography, MRI and scintigraphy. Clin Rheumatol 2008; 27: 983-989.
38. Gisondi P, Tinazzi I, El-Dalati G, et al. Lower limb enthesopathy in patients with psoriasis without clinical signs of arthropathy: a hospital-based case-control study. Ann Rheum Dis 2008; 67: 26-30.

39. De Filippis LG, Caliri A, Lo Gullo R, et al. Ultrasonography in the early diagnosis of psoriasis-associated enthesopathy. Int J Tissue React 2005; 27: 159-162.

40. Naredo E, Möller I, de Miguel E, et al. High prevalence of ultrasonographic synovitis and enthesopathy in patients with psoriasis without psoriatic arthritis: a prospective case-control study. Rheumatology (Oxford) 2011; 50: 1838-1848.

41. Freeston JE, Coates LC, Nam JL, et al. Is there subclinical synovitis in early psoriatic arthritis? A clinical comparison with gray-scale and power Doppler ultrasound. Arthritis Care Res (Hoboken) 2014; 66: 432-439.

42. Ash ZR, Tinazzi I, Gallego CC, et al. Psoriasis patients with nail disease have a greater magnitude of underlying systemic subclinical enthesiopathy than those with normal nails. Ann Rheum Dis 2012; 71: 553-561.

43. Benjamin M, McGonagle D. The enthesis organ concept and its relevance to the spondyloarthropathies. Adv Exp Med Biol 2009; 649: 57-70.

44. McGonagle D. Enthesitis: an autoinflammatory lesion linking nail and joint involvement in psoriatic disease. J Eur Acad Dermatol Venereol 2009; 23(Suppl 1): 9-13.

45. Sandre MK, Rohekar S. Psoriatic arthritis and nail changes: exploring the relationship. Semin Arthritis Rheum 2014; 44: 162-169.

46. Langenbruch A, Radtke MA, Krensel M, Jacobi A, Reich K, Augustin M. Nail involvement as a predictor of concomitant psoriatic arthritis in patients with psoriasis. Br J Dermatol 2014; 171: 1123-1128.

47. Villani AP, Rouzaud M, Sevrain M, et al. Symptoms dermatologists should look for in daily practice to improve detection of psoriatic arthritis in psoriasis patients: an expert group consensus. J Eur Acad Dermatol Venereol 2014; 28 Suppl 5: 27-32.

48. Dogra A, Arora AK. Nail psoriasis: the journey so far. Indian J Dermatol 2014; 59: 319-333. 